\title{
Effect of Facial Parameters on the Incidence of Rejection and Artifacts on the Video Head Impulse Test
}

\author{
Kwang-hyun Byun, $\mathrm{MD}^{1}$, Hye-soo Ryu, Aud ${ }^{2}$, Jae Yun Jung, $\mathrm{MD}, \mathrm{PhD}^{1}$, Ji Eun Choi, $\mathrm{MD}, \mathrm{PhD}^{1}$ and \\ Min Young Lee, $\mathrm{MD}, \mathrm{PhD}^{1}$ \\ ${ }^{1}$ Department of Otorhinolaryngology - Head and Neck Surgery, Dankook University College of Medicine, \\ Cheonan, Korea; and ${ }^{2}$ Department of Otorhinolaryngology - Head and Neck Surgery, Dankook University \\ Hospital, Cheonan, Korea
}

\begin{abstract}
- ABSTRACT -
Background and Objectives: The video head impulse test (vHIT) is a useful tool that uses a high-speed infrared video camera and an inertial accelerometer to quantify the vestibulo-ocular reflex. Despite advancements in recording technology, physicians should be aware of measurement artifacts induced by examiners. However, little is known about the impact of facial anatomy on artifacts. Therefore, we investigated how variance in facial anatomy affects measurement artifacts. Materials and Methods: This study enrolled nine subjects from November 2018 through November 2019. A digital camera was used to measure facial parameters, including head ratio, binocular spacing, nasofrontal angle, depth of nasion, and pupil ratio. Two examiners performed vHITs with ICS Impulse System (GN Otometrics). All impulses were assessed for artifacts, including rejection, notch during head impulse ( notch $_{\text {during }}$ ), notch after head impulse (notch ${ }_{\text {after }}$ ), and rebound. Results: The rejection rate was significantly correlated with depth of nasion, as measured by the experienced examiner, and with pupil ratio, as measured by the inexperienced examiner. notch during $_{\text {rate }}$ was significantly correlated with nasofrontal angle, as measured by the inexperienced examiner. Rebound rate was significantly correlated with head ratio, as measured by both examiners. Conclusions: Measurement artifacts were significantly correlated with head ratio, depth of nasion, nasofrontal angle, and pupil ratio. (J Clinical Otolaryngol 2021;32:101-110)
\end{abstract}

KEY WORDS: Artifacts; Face; Head impulse test.

\section{Introduction}

The video head impulse test (vHIT) is used to objectively measure the vestibulo-ocular reflex (VOR). The vHIT has several benefits over previous vestibular function tests, and evaluates more physiologic frequencies than the caloric test. Vertical semicircular canal function can also be measured with the vHIT. Advancements in imaging techniques have enabled detection of hidden corrective saccades (e.g., covert saccades), which are observed according to differences in eye and head velocities. $^{1-3)}$

The validity of the vHIT depends on the devices used to measure head and eye velocity. A gyroscope, accelerometer, and infrared camera are attached to goggles, which should in turn be firmly fixed to the patient's

Received: February 23, 2021 / Revised: April 19, 2021 / Accepted: July 5, 2021

Corresponding author: Ji Eun Choi, Department of Otorhinolaryngology-Head \& Neck Surgery, Dankook University Hospital, 201, Manghyang-ro, Dongnam-gu, Cheonan 31116, Korea

Tel: +82-41-550-3284 · Fax: +82-41-556-1090 - E-mail: garimung@gmail.com

Min Young Lee, Department of Otorhinolaryngology-Head \& Neck Surgery, Dankook University Hospital, 201, Manghyang-ro, Dongnam-gu, Cheonan 31116, Korea

Tel: +82-41-556-1090, Fax: +82-41-556-1090, E-mail: eyeglass210@gmail.com 
head to precisely measure head movement. To measure eye movement, the infrared camera should properly track the pupil. Therefore, calibration of the infrared camera with the pupil before actual testing is a very important step.

Artifacts are often observed on the vHIT, and can affect the test results in up to one-third of head impulse examinations. ${ }^{4)}$ These artifacts not only increase the test time, but also affect the test results because several artifacts resemble specific waveforms that occur pathologically. ${ }^{5-7)}$ The vHIT has a higher chance of showing artifacts than the search coil technique, which is the gold standard methodology for VOR evaluation. Causes of artifacts include error arising from the goggles, an incomplete eye-tracking algorithm, and a slow sampling rate. Common artifacts include rebound, mini-blink, oscillation, pseudo-saccades, and phase difference. ${ }^{4,8,9)}$ Rebound is an artifact caused by band slippage due to loosening of the strap or detached goggles. Mini-blink, pseudo saccade, oscillation might be caused by missed eye tracking system which leads to a unusual spiking graph on vHIT. Blinking the eye can cause unrelevant spikes on the chart and also spontaneous nystagmus can make a saccadic spike which can mislead the vHIT reading system. To reduce the incidence of artifacts, it is important to properly set up the vHIT to measure variables such as target distance, direction of impulse, velocity, and hand location. Experience in performing the test is also known to be important..$^{10,11)}$

Facial structures vary among individuals. The height of the nose and shape of the head affect how the goggles are supported. Moreover, anatomical variations in the eyes, such as the distance between them and pupil size, may affect the tracking of eye movement. Asian and Caucasian faces have some differences. Low nasal height can induce slippage of the goggles, and a weak eyelid elevator muscle may prevent proper eye tracking during the test. ${ }^{12,13)}$ However, few studies have demonstrated a correlation between artifact incidence and an- atomical variance in facial structures. Studies of Asian faces are especially necessary because due to marked facial structural variance, and because the vHIT was initially developed for Caucasian faces.

Therefore, in the present study, we assessed the incidence rates of several artifacts during the vHIT and their correlations with facial structure variance in subjects with normal vestibular function.

\section{Materials and Methods}

\section{Subjects}

Nine subjects ( $\geq 19$ years) were included in this study. The subjects had no past history of ear disease. We excluded subjects with a past history of dizziness, eye disease, cervical pathology, or ear surgery. Photographs were taken of all participants to measure their anatomical structures, and the vHIT was performed to analyze artifacts identified during the test. Our study followed the principles of the Declaration of Helsinki.

\section{Facial structure measurements}

To measure facial structures, each subject sat on a chair without back support. Photographs of the frontal and lateral aspects of the face, and top and bottom views, were taken with a X510HS camera (Canon, Tokyo, Japan). A 2.6-cm-long piece of tape was placed on each photograph and served as a scale. Adobe Photoshop CC (version 19.0; Adobe, Mountain View, CA, USA) was used to analyze five anatomic parameters. The head ratio was determined on the basis of the cephalic index, which is the ratio of the biparietal diameter to the occipitofrontal diameter (OFD). The biparietal diameter is the maximum width of the skull connecting both parietal bones, and the OFD is the distance between the eyebrow and occipital bone (Fig. 1A). Binocular spacing was calculated as the ratio between the interpupillary and bizygomatic distances (Fig. 1B). The nasofrontal angle is the obtuse angle between 
the lines tangent to the glabella and rhinion, with both lines originating from the nasion (Fig. 1C). The depth of the nasion was defined as the length of the vertical line that connects the rhinion and the imaginary line that connects the glabella and rhinion (Fig. 1C). The pupil ratio, defined as the ratio of the height of the pupil to the width of the exposed cornea, which was measured in the right eye (Fig. 1D).

\section{The video head impulse test (vHIT) and arti-} facts

The vHIT was performed with the ICS Impulse System (GN Otometrics, Taastrup, Denmark). Subjects were instructed to gaze at the target, which was placed 1 meter away from them. After device setup and calibration, the subject performed head impulses at a velocity of $150^{\circ}-200^{\circ} / \mathrm{s}$ to facilitate evaluation of the lateral semicircular canals, and impulses at a velocity of $100^{\circ}-150^{\circ} / \mathrm{s}$ to facilitate evaluation of the vertical semicircular canals. Two different examiners evaluated each subject, using two sets of tests. One examiner was inexperienced (a right-handed man who had performed $<100$ tests), and the other was experienced (a right-handed woman who had performed $>500$ tests).
The two examiners performed the two sets of tests in a random order. The examiner placed both hands on the chin of the subject to guide the head impulse for evaluation of the lateral semicircular canal; the examiner's right hand was placed on the vertex, the left hand was placed on the chin, and the head was rotated $35^{\circ}-45^{\circ}$ to each side.

In the six semicircular canals (left lateral, right lateral, left anterior, right posterior, right anterior, and left posterior) examined, four different artifacts were observed: rejection, notch during head impulse (notchduring), notch after head impulse (notch after $_{\text {) }}$, and rebound (Fig. 2). Rejection was automatically determined by the algorithm of the ICS Impulse Device; this occurs when the sampling rate is low (i.e., $<219$ frames/s). A notch was noted when more than two peaks in eye velocity were present. notch during was classified as a velocity peak greater than $25 \%$ s during head movement and after the maximal eye-velocity peak (Fig. 2A). notch $_{\text {after }}$ was classified as a velocity peak greater than $50 \%$ s after head movement (Fig. 2B) [7]. Rebound was classified as counter-impulse eye movement during the latter part of head movement with a velocity greater than $25 \% \mathrm{~s}$ (Fig. 2C). The rejection ratio was defined as the number

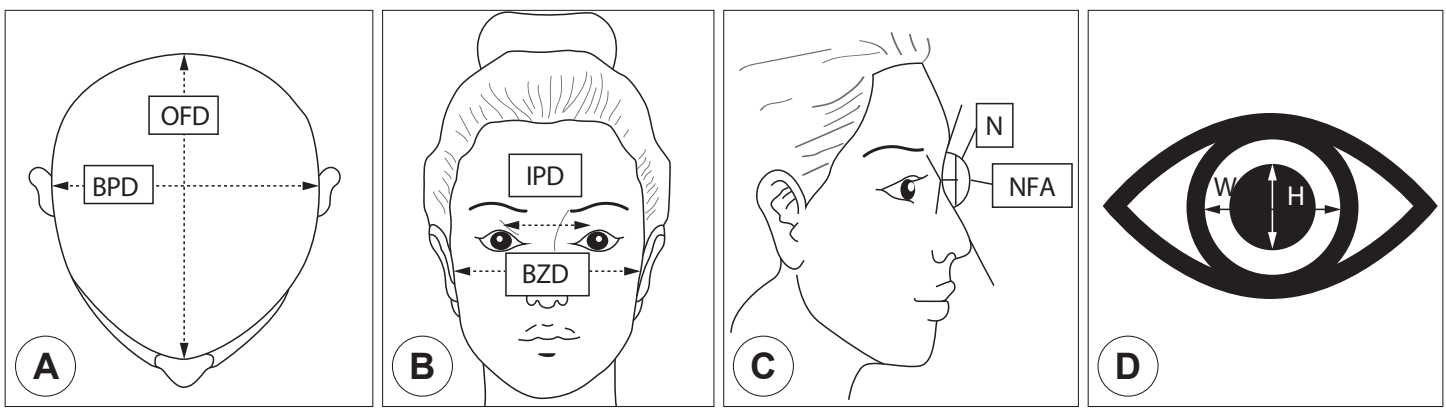

Fig. 1. Illustrations of facial parameters. (A) The head ratio is defined as the ratio between the biparietal diameter (BDP) and occipitofrontal diameter (OFD) of the head. BDP is the maximum width (side to side) of the head, and OFD is the maximum length (front to back) of the head (head ratio (\%)=BPD/OFD $\times 100$ ).(B) Binocular spacing is defined as the ratio between the interpupillary distance (IPD) and bizygomatic distance. IPD is the distance between the pupils. Bizygomatic distance (BZD) is the maximal distance between the most lateral points on the zygomatic arches (binocular spacing (\%)=IPD/BZD×100). (C) The nasofrontal angle (NFA) is the obtuse angle between the line tangent to the glabella and the line tangent to the rhinion, with both lines originating from the nasion. The depth of nasion (N) is the shortest distance between the nasion and the line from the glabella to the rhinion. (D) The pupil ratio is defined as the ratio of the height $(\mathrm{H})$ of pupil to the width $(\mathrm{W})$ of the exposed cornea (cornea ratio $(\%)=H W \times 100)$. 


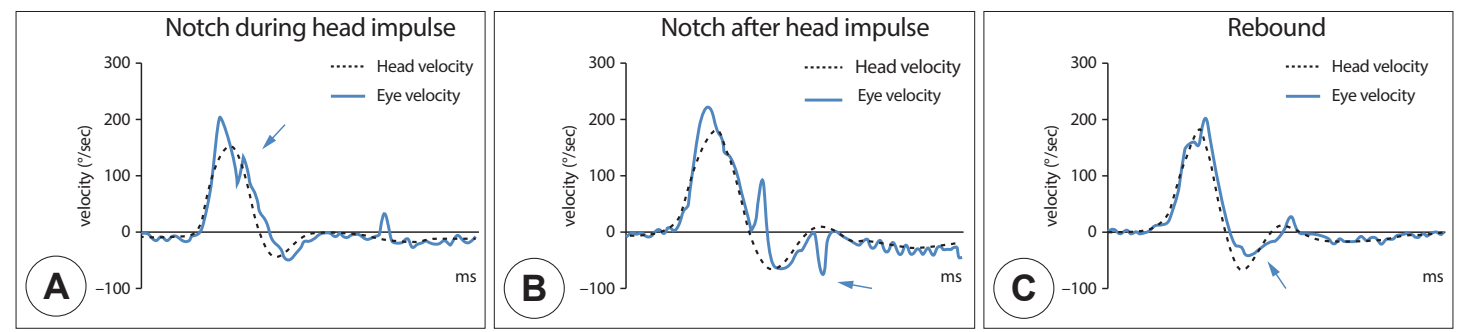

Fig. 2. Example artifacts. Each type of artifact is illustrated by the graphs (the x-axis shows time, and the $y$-axis shows head and eye velocity). The black and blue lines indicate head and eye velocity, respectively. The graphs show traces of artifact-related eye and head movements. (A) Notch during head impulse (arrow) is defined as the change in direction of the eye with velocity greater than $25 \%$ during head impulse. (B) Notch after head impulse (arrow) is defined as the change in direction of the eye with velocity greater than $50 \% / s$ after head impulse. Showing a sharp direction changing peak on the graph. (C) Rebound (arrow) is defined as eye movement in the opposite direction, with velocity greater than $25 \%$ at the end of head impulse; this is caused by the head inadvertently moving back.

of rejections divided by the total number of impulses. notch $_{\text {during, }}$, notch ${ }_{\text {after }}$, and the rebound ratio was defined as the number of each artifact type divided by the total number of impulses that were not rejected.

\section{Statistics}

IBM SPSS statistics software (version 20.0; IBM, Armonk, NY, USA) was used to perform the statistical analysis. The Wilcoxon signed-rank test or paired t-test was used to compare the number of artifacts detected between the two examiners. Pearson or Spearman rank correlation was used to evaluate the correlation between variations in facial anatomy and artifacts. p-values less than 0.05 were considered statistically significant.

\section{Results}

The mean age of the subjects was 30.2 years (Table 1). All subjects showed vHIT gain of more than 0.6, with no corrective saccade for any of the six semicircular canals.

The incidence rates of the four different artifacts in all six semicircular canals were compared between the two examiners. The experienced examiner had a rejection rate of $25.2 \%$, notch during $_{\text {rate of }} 29.6 \%$, notch after $_{\text {rate of }}$ $4.28 \%$, and rebound rate of $52.5 \%$. The inexperienced
Table 1. Demographics and facial anatomy parameters

\begin{tabular}{lc}
\hline Enrolled number & 9 \\
Age (years) & $30.2 \pm 3.9$ \\
Sex (female:male) & $3: 6$ \\
Head ratio (\%) & $89.7 \pm 4.4$ \\
Binocular spacing (\%) & $45.6 \pm 1.6$ \\
Pupil ratio (\%) & $70.7 \pm 13.2$ \\
Depth of nasion $(\mathrm{cm})$ & $1.04 \pm 0.17$ \\
Nosofrontal angle $\left({ }^{\circ}\right)$ & $134.4 \pm 5.7$ \\
\hline
\end{tabular}

examiner had a rejection rate of $38.6 \%$, notch during rate of $25.2 \%$, notch $_{\text {after }}$ rate of $6.6 \%$, and rebound rate of $50.4 \%$ (Fig. 3). For both examiners, the notch ${ }_{\text {after }}$ ratio was low $(<10 \%)$ and the rebound ratio was high $(>50 \%)$. The rejection rate was statistically higher for the inexperienced examiner compared with the experienced examiner $(\mathrm{t}(8)=-2.338, \mathrm{p}=0.048)$.

In the semicircular canal-specific analyses, the two examiners had significantly different rejection rates for the left lateral canal $(\mathrm{t}(8)=-2.449, \mathrm{p}=0.040)$ and left anterior canal $(\mathrm{t}(8)=-2.630, \mathrm{p}=0.030)$ (Table 2). The inexperienced examiner had a high notch ${ }_{\text {after }}$ ratio $(>10 \%)$ for the bilateral horizontal canal, and the left-

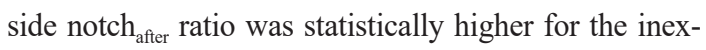
perienced examiner compared with the experienced examiner $(Z=2.023, p=0.043)$. These results suggest that a higher rejection rate and higher incidence of certain 


\section{Artifact rates}

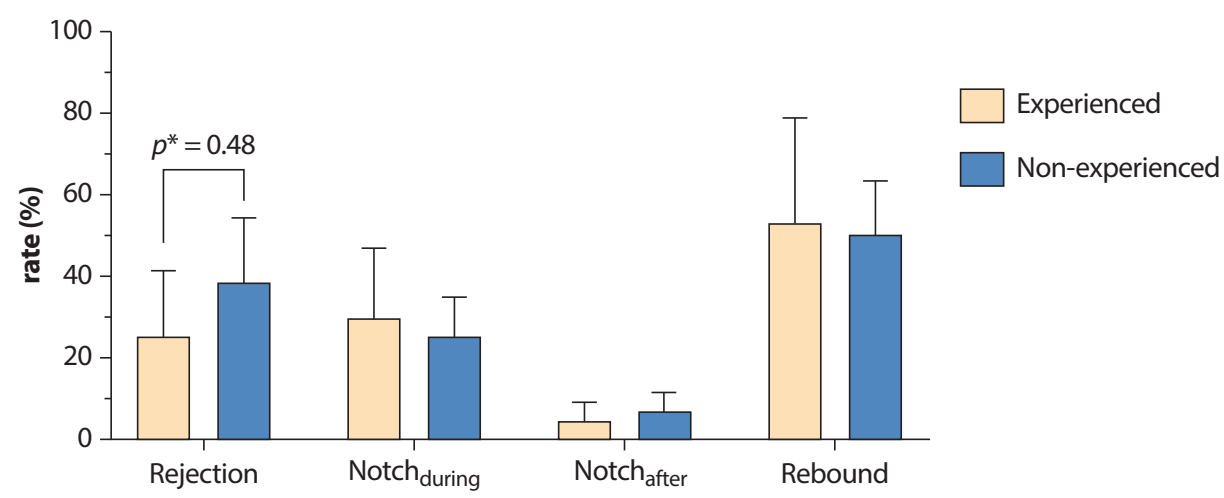

Fig. 3. Comparison of artifact rates in all canals. Bar graphs show the mean and range values of the artifact rates. Each artifact rate is the mean value of all six canals. ${ }^{*}$ Indicates a statistically significant difference, as determined with the paired t-test.

Table 2. Comparisons of artifact rates for each canal between two examiners

\begin{tabular}{|c|c|c|c|c|}
\hline Artifact rates & Canals & Experienced & Non-experienced & p-value \\
\hline \multirow[t]{6}{*}{ Rejection } & $\mathrm{LL}$ & $21.6 \pm 8.7 \%$ & $41.6 \pm 20.0 \%$ & $0.040^{*}$ \\
\hline & $\mathrm{RL}$ & $24.8 \pm 12.3 \%$ & $33.2 \pm 20.9 \%$ & $0.390 *$ \\
\hline & LA & $21.4 \pm 10.1 \%$ & $35.0 \pm 15.7 \%$ & $0.030 *$ \\
\hline & $\mathrm{RP}$ & $16.2 \pm 18.9 \%$ & $39.6 \pm 27.7 \%$ & $0.063^{*}$ \\
\hline & RA & $34.9 \pm 24.4 \%$ & $38.7 \pm 18.6 \%$ & $0.611^{*}$ \\
\hline & LP & $32.0 \pm 24.4 \%$ & $43.7 \pm 26.2 \%$ & $0.553 \dagger$ \\
\hline \multirow{6}{*}{$\begin{array}{l}\text { Notch during head } \\
\text { impulse }\end{array}$} & LL & $12.6 \pm 17.2 \%$ & $26.7 \pm 19.0 \%$ & $0.133^{*}$ \\
\hline & $R L$ & $19.9 \pm 15.9 \%$ & $18.3 \pm 19.1 \%$ & $0.846^{*}$ \\
\hline & LA & $42.7 \pm 26.2 \%$ & $24.3 \pm 22.8 \%$ & $0.105^{*}$ \\
\hline & $\mathrm{RP}$ & $36.7 \pm 32.6 \%$ & $29.8 \pm 24.3 \%$ & $0.675^{*}$ \\
\hline & RA & $24.0 \pm 18.4 \%$ & $21.6 \pm 13.0 \%$ & $0.712^{*}$ \\
\hline & LP & $42.0 \pm 32.9 \%$ & $30.3 \pm 29.6 \%$ & $0.356^{*}$ \\
\hline \multirow{6}{*}{$\begin{array}{l}\text { Notch after head } \\
\text { impulse }\end{array}$} & LL & $6.1 \pm 9.6 \%$ & $12.4 \pm 20.5 \%$ & $0.043 \dagger$ \\
\hline & $R L$ & $7.8 \pm 9.7 \%$ & $11.0 \pm 12.7 \%$ & $0.577^{*}$ \\
\hline & LA & $1.3 \pm 2.7 \%$ & $4.8 \pm 9.6 \%$ & $0.465+$ \\
\hline & $\mathrm{RP}$ & $0.8 \pm 2.3 \%$ & $4.6 \pm 5.9 \%$ & $0.143^{*}$ \\
\hline & RA & $7.2 \pm 9.5 \%$ & $2.6 \pm 4.1 \%$ & $0.230^{*}$ \\
\hline & LP & $2.4 \pm 5.5 \%$ & $4.0 \pm 4.7 \%$ & $0.582^{*}$ \\
\hline \multirow[t]{6}{*}{ Rebound } & $\mathrm{LL}$ & $68.4 \pm 25.4 \%$ & $54.1 \pm 14.8 \%$ & $0.232 *$ \\
\hline & $\mathrm{RL}$ & $74.1 \pm 14.5 \%$ & $71.3 \pm 23.5 \%$ & $0.798^{*}$ \\
\hline & LA & $21.8 \pm 24.9 \%$ & $38.6 \pm 33.6 \%$ & $0.161^{*}$ \\
\hline & RP & $43.6 \pm 29.6 \%$ & $47.7 \pm 25.7 \%$ & $0.441^{*}$ \\
\hline & RA & $44.9 \pm 32.8 \%$ & $25.8 \pm 25.0 \%$ & $0.137^{*}$ \\
\hline & LP & $62.7 \pm 35.8 \%$ & $65.2 \pm 25.2 \%$ & $0.822 *$ \\
\hline
\end{tabular}

* paired t-test, †Wilcoxon signed-rank test.

LL: left lateral, RL: right lateral, LA: left anterior, RP: right posterior, RA: right anterior, LP: left posterior. 
artifacts would be expected for inexperienced examiners compared with experienced examiners.

Table 3 is shows the correlation between facial parameters and artifacts in all six semicircular canals; the notch $_{\text {after }}$ ratio was excluded owing to its low incidence. The binocular spacing rate was not correlated with facial parameters. For the inexperienced examiner, three facial parameters were correlated with artifacts: the head ratio and rebound rate were positively correlated $(\mathrm{r}=0.758, \mathrm{p}=0.018)$; the nasofrontal angle and notch during $_{\text {. }}$ rate were negatively correlated $(r=-0.669, \mathrm{p}=0.049)$; and the pupil ratio and rejection rate were negatively correlated $(r=-0.718, p=0.029)$. For the experienced examiner, there was a single positive significant correlation, between the depth of nasion and rejection rate $(\mathrm{r}=0.722, \mathrm{p}=0.028)$.

In the semicircular canal-specific analyses, positive correlations between the head ratio and rebound rate in the right posterior semicircular canal were seen for both the experienced and inexperienced examiner $(r=0.697$, $\mathrm{p}=0.037$; and $\mathrm{r}=0.757, \mathrm{p}=0.018$, respectively). The same correlation in the left posterior semicircular canal was also seen for the inexperienced examiner $(r=0.818$, $\mathrm{p}=0.007$ ) (Table 4). A positive correlation between the depth of nasion and rejection rate in the left posterior semicircular canal was seen for the experienced examiner $(\mathrm{r}=0.815, \mathrm{p}=0.007)$, as well as between the pupil ratio and notchduring rate $(r=0.693, p=0.039)$. A negative correlation between the pupil ratio and the rejection rate in the right lateral semicircular canal was seen for the inexperienced examiner $(r=-0.847, \mathrm{p}=0.004)$. Correlations between facial parameters and artifacts were mostly observed in the posterior canal (four in the posterior semicircular canal and one in the lateral semicircular canal). In other words, we speculate that facial structure-related artifacts usually occur in the posterior semicircular canal.

\section{Discussion}

In the present study, we compared artifact rates observed during the vHIT between experienced and inexperienced examiners. As expected, different rejection rates were observed, with a higher rejection rate for the inexperienced examiner. However, we observed no differences in other artifacts (Fig. 3). An increased rejection rate may increase test time, but would not affect the test outcomes because rejected waves would be excluded. In analyses of the six semicircular canals, differences in artifacts in the left semicircular canals (left

Table 3. Correlation between facial parameters and artifacts for total canals

\begin{tabular}{|c|c|c|c|c|c|c|c|}
\hline \multirow{2}{*}{ Variables } & & \multicolumn{3}{|c|}{ Experienced examiner } & \multicolumn{3}{|c|}{ Non-experienced examiner } \\
\hline & & Rejection & Notch $_{\text {during }}$ & Rebound & Rejection & Notch $_{\text {during }}$ & Rebound \\
\hline \multirow[t]{2}{*}{ Head ratio } & $r$ & -0.136 & -0.157 & 0.275 & 0.178 & 0.354 & 0.758 \\
\hline & $\mathrm{p}$ & 0.727 & 0.686 & 0.474 & 0.646 & 0.350 & 0.018 \\
\hline \multirow[t]{2}{*}{ Binocular spacing } & $r$ & -0.048 & 0.138 & -0.176 & -0.436 & -0.178 & -0.347 \\
\hline & $p$ & 0.903 & 0.722 & 0.650 & 0.241 & 0.646 & 0.360 \\
\hline \multirow[t]{2}{*}{ Depth of nasion } & r & 0.722 & -0.101 & 0.070 & 0.083 & 0.480 & 0.105 \\
\hline & $p$ & 0.028 & 0.796 & 0.858 & 0.831 & 0.191 & 0.789 \\
\hline \multirow[t]{2}{*}{ Nasofrontal angle } & r & -0.666 & 0.146 & -0.150 & -0.406 & -0.669 & -0.435 \\
\hline & $p$ & 0.050 & 0.707 & 0.700 & 0.278 & 0.049 & 0.242 \\
\hline \multirow[t]{2}{*}{ Pupil ratio } & $r$ & -0.171 & 0.506 & -0.462 & -0.718 & 0.044 & -0.378 \\
\hline & $p$ & 0.659 & 0.164 & 0.211 & 0.029 & 0.911 & 0.316 \\
\hline
\end{tabular}

Pearson correlation analysis was used. 
Kwang-hyun Byun, et al : Facial Structure and Artifacts on the Video Head Impulse Test

Table 4. Correlation between facial parameters and artifacts for each canal

\begin{tabular}{|c|c|c|c|c|c|c|c|}
\hline \multirow{2}{*}{\multicolumn{2}{|c|}{ Canal }} & \multicolumn{2}{|c|}{ Rejection } & \multicolumn{2}{|c|}{ Notch $_{\text {during }}$} & \multicolumn{2}{|c|}{ Rebound } \\
\hline & & Exp & Non-exp & Exp & Non-exp & Exp & Non-exp \\
\hline \multicolumn{8}{|c|}{ Head ratio } \\
\hline \multirow[t]{2}{*}{ LL } & $r$ & 0.170 & -0.180 & 0.592 & 0.231 & -0.272 & -0.612 \\
\hline & $\mathrm{p}$ & 0.661 & 0.643 & 0.093 & 0.549 & 0.479 & 0.080 \\
\hline \multirow[t]{2}{*}{ RL } & $r$ & 0.271 & -0.142 & 0.037 & -0.143 & 0.475 & 0.154 \\
\hline & $\mathrm{p}$ & 0.481 & 0.715 & 0.924 & 0.713 & 0.196 & 0.693 \\
\hline \multirow[t]{2}{*}{ LA } & $r$ & -0.060 & 0.542 & -0.589 & 0.111 & -0.120 & 0.467 \\
\hline & $\mathrm{p}$ & 0.879 & 0.131 & 0.095 & 0.776 & 0.758 & 0.205 \\
\hline \multirow[t]{2}{*}{ RP } & $r$ & 0.639 & 0.151 & -0.152 & 0.353 & 0.697 & 0.757 \\
\hline & $\mathrm{p}$ & 0.064 & 0.699 & 0.695 & 0.352 & 0.037 & 0.018 \\
\hline \multirow[t]{2}{*}{ RA } & $r$ & -0.433 & 0.226 & -0.301 & -0.204 & -0.016 & 0.380 \\
\hline & $\mathrm{p}$ & 0.244 & 0.558 & 0.432 & 0.598 & 0.967 & 0.314 \\
\hline \multirow[t]{2}{*}{ LP } & $r$ & -0.349 & 0.241 & 0.103 & 0.370 & 0.434 & 0.818 \\
\hline & $p$ & 0.358 & 0.532 & 0.792 & 0.327 & 0.244 & 0.007 \\
\hline \multicolumn{8}{|c|}{ Depth of nasion } \\
\hline \multirow[t]{2}{*}{ LL } & $r$ & 0.211 & 0.613 & -0.087 & 0.078 & -0.124 & -0.086 \\
\hline & $\mathrm{p}$ & 0.585 & 0.079 & 0.824 & 0.841 & 0.751 & 0.826 \\
\hline \multirow[t]{2}{*}{ RL } & r & 0.353 & 0.307 & -0.008 & 0.502 & 0.315 & 0.294 \\
\hline & $\mathrm{p}$ & 0.351 & 0.422 & 0.983 & 0.168 & 0.409 & 0.443 \\
\hline \multirow[t]{2}{*}{ LA } & $r$ & -0.157 & 0.056 & 0.334 & 0.520 & 0.086 & -0.489 \\
\hline & $\mathrm{p}$ & 0.687 & 0.887 & 0.379 & 0.151 & 0.825 & 0.181 \\
\hline \multirow[t]{2}{*}{ RP } & $r$ & 0.075 & -0.213 & -0.520 & 0.098 & 0.237 & 0.210 \\
\hline & $\mathrm{p}$ & 0.847 & 0.582 & 0.151 & 0.801 & 0.538 & 0.588 \\
\hline \multirow[t]{2}{*}{ RA } & $r$ & 0.453 & 0.120 & 0.059 & -0.202 & -0.046 & 0.149 \\
\hline & $\mathrm{p}$ & 0.221 & 0.758 & 0.881 & 0.602 & 0.906 & 0.703 \\
\hline \multirow[t]{2}{*}{ LP } & $r$ & 0.815 & -0.128 & 0.036 & 0.355 & 0.234 & 0.395 \\
\hline & $\mathrm{p}$ & 0.007 & 0.743 & 0.927 & 0.348 & 0.544 & 0.293 \\
\hline \multicolumn{8}{|c|}{ Nasofrontal angle } \\
\hline \multirow[t]{2}{*}{ LL } & $r$ & -0.315 & -0.501 & -0.236 & -0.419 & 0.477 & -0.005 \\
\hline & $p$ & 0.410 & 0.169 & 0.542 & 0.261 & 0.195 & 0.990 \\
\hline \multirow[t]{2}{*}{ RL } & $r$ & -0.455 & -0.342 & 0.143 & -0.115 & -0.178 & -0.168 \\
\hline & $p$ & 0.218 & 0.368 & 0.713 & 0.769 & 0.647 & 0.666 \\
\hline \multirow[t]{2}{*}{ LA } & $r$ & 0.000 & -0.354 & -0.114 & -0.326 & -0.113 & 0.023 \\
\hline & $p$ & 1.000 & 0.350 & 0.769 & 0.392 & 0.773 & 0.952 \\
\hline \multirow[t]{2}{*}{ RP } & $r$ & -0.388 & -0.187 & 0.403 & -0.440 & -0.304 & -0.371 \\
\hline & $p$ & 0.302 & 0.629 & 0.283 & 0.236 & 0.427 & 0.326 \\
\hline \multirow[t]{2}{*}{ RA } & $r$ & -0.343 & -0.147 & -0.212 & 0.232 & -0.047 & -0.234 \\
\hline & $\mathrm{p}$ & 0.366 & 0.706 & 0.583 & 0.548 & 0.905 & 0.544 \\
\hline \multirow[t]{2}{*}{ LP } & $r$ & -0.580 & -0.277 & 0.198 & -0.493 & -0.390 & -0.621 \\
\hline & $p$ & 0.101 & 0.470 & 0.610 & 0.178 & 0.300 & 0.074 \\
\hline
\end{tabular}


Table 4. Continued

\begin{tabular}{|c|c|c|c|c|c|c|c|}
\hline \multirow{2}{*}{\multicolumn{2}{|c|}{ Canal }} & \multicolumn{2}{|c|}{ Rejection } & \multicolumn{2}{|c|}{ Notch $_{\text {during }}$} & \multicolumn{2}{|c|}{ Rebound } \\
\hline & & Exp & Non-exp & Exp & Non-exp & Exp & Non-exp \\
\hline \multicolumn{8}{|c|}{ Pupil ratio } \\
\hline \multirow[t]{2}{*}{ LL } & $r$ & 0.473 & -0.602 & -0.086 & -0.040 & -0.028 & 0.142 \\
\hline & $\mathrm{p}$ & 0.198 & 0.087 & 0.827 & 0.919 & 0.942 & 0.716 \\
\hline \multirow[t]{2}{*}{ RL } & $r$ & 0.236 & -0.847 & 0.628 & -0.195 & -0.314 & -0.084 \\
\hline & $\mathrm{p}$ & 0.542 & 0.004 & 0.070 & 0.614 & 0.410 & 0.831 \\
\hline \multirow[t]{2}{*}{ LA } & $r$ & 0.332 & -0.342 & -0.091 & 0.664 & -0.572 & -0.279 \\
\hline & $\mathrm{p}$ & 0.383 & 0.367 & 0.817 & 0.051 & 0.108 & 0.467 \\
\hline \multirow[t]{2}{*}{$\mathrm{RP}$} & $r$ & 0.128 & -0.373 & 0.265 & -0.279 & -0.088 & -0.100 \\
\hline & $p$ & 0.743 & 0.323 & 0.490 & 0.467 & 0.823 & 0.798 \\
\hline \multirow[t]{2}{*}{ RA } & $r$ & -0.380 & -0.337 & 0.016 & -0.072 & -0.518 & -0.488 \\
\hline & $p$ & 0.313 & 0.375 & 0.967 & 0.855 & 0.153 & 0.183 \\
\hline \multirow[t]{2}{*}{ LP } & $r$ & -0.481 & -0.586 & 0.693 & -0.011 & -0.438 & -0.229 \\
\hline & $\mathrm{p}$ & 0.189 & 0.097 & 0.039 & 0.977 & 0.238 & 0.553 \\
\hline
\end{tabular}

Pearson or spearman correlation analysis was used.

anterior and left lateral) were observed between the two examiners, suggesting that differences in the dominant head impulse led to artifacts in the examinations performed by the inexperienced examiner.

In the analysis of all six semicircular canals, four correlations between facial parameters and artifacts were observed: one for the experienced examiner and three for the inexperienced examiner. As the depth of nasion increased, the rejection rate of the experienced examiner increased. Three correlations between artifacts and facial parameters were seen for the inexperienced examiner (one positive and two negative). As the pupil ratio decreased, the rejection rate increased. A smaller nasofrontal angle was associated with a higher notch incidence. In addition, the head ratio increased as the rebound incidence increased. Other than the rejection rate, correlations between artifacts and parameters were observed only for the inexperienced examiner. These results suggest that less experience may lead to facial parameter-related artifacts. Presence of notch is related to a small nasofrontal angle which could lead to improper wearing of goggles and mis-track the pupil to blink or move, and rebound is related to a round head shape which could have more soft tissue portion around the skull to let the bandage slip easily. ${ }^{14)}$ Owing to the combination of differences in both the artifacts present and the correlation outcomes, the results of the inexperienced examiner showed more artifacts and correlations between artifacts and facial parameters without an increase in the incidence of artifacts. However, there have been some issues on goggle slippage due to strap tightness and Asians having a lower nasal dorsum is a fact we know. There should be larger study group and experienced vHIT performer only study for further research. $^{15,16)}$

In the analysis of each of the six semicircular canals, positive correlations between the head ratio and rebound rate in the right anterior canal were seen for both examiners. This suggests that, regardless of experience, examiners should be aware of the possibility of rebound artifacts in the right-side (i.e., dominant hand) posterior canal in subjects with a high head ratio.

Recent, publications have emphasized quality of the impulse over its quantity during vHIT. Wenzel et al. have demonstrated that with high speed no artifact impulse adequate data have acquired even with low num- 
ber of impulses in pediatric population. ${ }^{17)}$ Trinidad-Ruiz $\mathrm{G}$ et al. have suggested removing artifacts among vHIT samples to increase the precision. ${ }^{18)}$ As such quality of head impulse should be adequate for vHIT. For this reason, identification and correction of artifacts in each impulse are being more emphasized.

Small technical adjustments could reduce the rejection rate associated with pupil size. The experienced examiner used techniques to fix the upper eyelid and thus improve pupil tracking, but the inexperienced examiner did not. However, involuntary or voluntary forced opening of the upper lid may increase the incidence of mini-blink, which could in turn result in a notch during $_{\text {g }}$ artifact (in unrejected eye waves) during evaluation by an experienced examiner. But, Asian upper eyelids have an unique feature which has prominent submuscular fibro-adipose tissue around orbicularis oculi compared to caucasians. 12 As we consider this point, in Asian population, it might be helpful to fix the eyelids during the vHIT to open the whole eyefield. The rebound rate was relatively high $(>50 \%)$ for both examiners. Regardless of examiner experience, larger head width increased the rebound rate; increased hand force of the dominant hand may be necessary in such cases, but this could increase rebound. The nasofrontal angle was another facial parameter that was correlated with the rebound rate, but the results of the analyses of all canals and each separate canal were inconsistent; further analysis with a larger number of subjects is required. The limitations of the current study include the small number of subjects $(\mathrm{N}=9)$, the possibility that examiner experience and presence of artifacts are highly correlated, and the possibility of artifacts in rejected waves. Despite these limitations, the results indicated that artifacts may be related to facial parameters. Further research is needed with a greater number of subjects, minimization of the effects of other variables, and a focus on facial parameters that may affect the incidence of artifacts.

\section{Acknowledgements}

The English in this document has been checked by at least two professional editors, both native speakers of English. For a certificate, please see: http://www.textcheck.com/certificate/ HdZGQU.

\section{Funding Information}

Not applicable.

\section{Conflicts of Interest}

No potential conflict of interest relevant to this article was reported.

\section{ORCID}

Kwang-hyun Byun, https://orcid.org/0000-0002-5076-6026 Hye-soo Ryu, https://orcid.org/0000-0003-4954-3877 Jae Yun Jung, http://orcid.org/0000-0002-1870-0748 Ji Eun Choi, https://orcid.org/0000-0001-8105-813X Min Young Lee, https://orcid.org/0000-0002-6860-8042

\section{Author Contribution}

Conceptualization: Jung JY, Choi JE, Lee MY. Formal analysis: Byun KH, Choi JE, Lee MY. Methodology: Byun KH, Ryu HS. Investigation: Jung JY, Choi JE, Lee MY. Writing - original draft: Byun KH, Choi JE, Lee MY. Writing - review \& editing: Byun KH, Ryu HS, Jung JY, Choi JE, Lee MY

\section{Ethics Approval}

Our study followed the principles of the Declaration of Helsinki.

\section{References}

1) Ulmer E, Chays A. Curthoys and halmagyi head impulse test: an analytical device. Ann Otolaryngol Chir Cervicofac 2005;122(2):84-90.

2) MacDougall HG, Weber KP, McGarvie LA, Halmagyi GM, Curthoys IS. The video head impulse test: diagnostic accuracy in peripheral vestibulopathy. Neurology 2009;73(14): 1134-41.

3) MacDougall HG, McGarvie LA, Halmagyi GM, Curthoys IS, Weber KP. Application of the video head impulse test to detect vertical semicircular canal dysfunction. Otol Neurotol 2013;34(6):974-9.

4) Mantokoudis G, Tehrani ASS, Kattah JC, Eibenberger K, Guede CI, Zee DS, et al. Quantifying the vestibulo-ocular reflex with video-oculography: nature and frequency of artifacts. Audiol Neurotol 2015;20(1):39-50.

5) McGarvie LA, Martinez-Lopez M, Burgess AM, MacDougall HG, Curthoys IS. Horizontal eye position affects 
measured vertical VOR gain on the video head impulse test. Front Neurol 2015;6:58.

6) Patterson JN, Bassett AM, Mollak CM, Honaker JA. Effects of hand placement technique on the video head impulse test (vHIT) in younger and older adults. Otol Neurotol 2015; 36(6):1061-8

7) Mantokoudis G, Tehrani ASS, Xie L, Eibenberger K, Eibenberger B, Roberts D, et al. The video head impulse test during post-rotatory nystagmus: physiology and clinical implications. Exp Brain Res 2016;234(1):277-86.

8) Mantokoudis G, Saber Tehrani AS, Wozniak A, Eibenberger K, Kattah JC, Guede CI, et al. Impact of artifacts on VOR gain measures by video-oculography in the acute vestibular syndrome. J Vestib Res 2016;26(4):375-85.

9) Heuberger M, Grill E, Sağlam M, Ramaioli C, Müller M, Strobl R, et al. Usability of the video head impulse test: lessons from the population-based prospective KORA study. Front Neurol 2018;9:659.

10) Halmagyi GM, Curthoys IS. A clinical sign of canal paresis. Arch Neurol 1988;45(7):737-9.

11) Halmagyi GM. Diagnosis and management of vertigo. Clini Med 2005;5(2):159-65.
12) Saonanon P. Update on Asian eyelid anatomy and clinical relevance. Curr Opin Ophthalmol 2014;25(5):436-42.

13) McCurdy JA Jr. The Asian nose: augmentation rhinoplasty with L-shaped silicone implants. Facial Plast Surg 2002; 18(4):245-52.

14) Halmagyi GM, Chen L, MacDougall HG, Weber KP, McGarvie LA, Curthoys IS. The video head impulse test. Front Neurol 2017;8:258.

15) Suh MW, Park JH, Kang SI, Lim JH, Park MK, Kwon SK. Effect of goggle slippage on the video head impulse test outcome and its mechanisms. Otol Neurotol 2017;38(1):102-9.

16) Jang YJ, Yu MS. Rhinoplasty for the Asian nose. Facial Plast Surg 2010;26(2):93-101.

17) Wenzel A, Hülse R, Thunsdorff C, Rotter N, Curthoys I. Reducing the number of impulses in video head impulse testing - it's the quality not the numbers. Int J Pediatr Otorhinolaryngol 2019;125:206-11.

18) Trinidad-Ruiz G, Rey-Martinez J, Matiño-Soler E, Batuecas-Caletrio A, Martin-Sanz E, Perez-Fernandez N. Relevance of artifact removal and number of stimuli for video head impulse test examination. Ear Hear 2020;41(5):1397-406. 